\title{
The Global Impact of the Mo-99 Shortage
}

\author{
Michael AB Naafs* \\ Dutch Internist-Endocrinologist, International Health Consultancy, Netherlands
}

Received: May 7, 2018; Published: May 25, 2018

*Corresponding author: Michael AB Naafs, Dutch Internist-Endocrinologist, Health Consultant at Naafs, International Health Consultancy, Rhodoslaan 20 , and $7577 \mathrm{KN}$, Oldenzaal, The Netherlands

\begin{abstract}
In this mini-review, the history and discovery of molybdene, its use and production, waste problem and alternative cyclotron medical isotope production are discussed. Definite reactor closing and extensive nuclear construction works in 2018 will comprise global medical isotope supply further.
\end{abstract}

\section{Introduction}

Recently, various reports appeared concerning a global threatening shortage of molybdene (1-4).Medical diagnostic imaging techniques using tehcnetium-99m (99mTC) account for approximately of all nuclear medicine procedures, representing 30-40 million examinations worldwide every year. Technetium 99 is derived from molybdene-99. Disruptions in the supply chain of these medical isotopes, which have half-lives of 66 hours for molybdene-99 (99Mo) and only 6 hours for $99 \mathrm{mTc}$, and thus must be produced continuously, can lead to cancellations or delays in important medical testing services. Supply reliability has been challenged over the past decade due to unexpected shutdowns and extended refurbishment periods at some of the $99 \mathrm{Mo}$-producing research reactors and processing facilities. These shutdowns have at times created conditions for extended global supply shortages (e.g., 2009-2010) -(2,3). Nearly 95 percent of the world's supply of molybdenum-99-99m is produced by irradiating targets, typically a solid plate containing uranium clad in aluminum, in seven research reactors located in Australia, Canada, Argentina, Brazil Mexico, Europe, and South-Africa.

This isotope has not been produced in the U.S. since the late 1980's. Molybdenum-99 and technecium-99 are distributed through an international supply chain on a weekly or more frequent basis. Such speedy delivery is essential because molybdenum and technetium-99m have short halve-lives and therefore cannot be stockpiled. As the out of time reactor in Canada shuts down permanently at the end of March 2018, the U.S will lose a supplier of last resort. There is a greater than $50 \%$ change that severe shortages of Mo-99 and Tc-99m after closing the NRU reactor at Chalk River, Canada [1-4]. For that reason, the University of Missouri Reserch
Reactor, with its partners, Nordien and General Atomics, filed for NRC in March 2017, approval to start U.S. production of medical isotopes [5]. The start of the renovation in 2018 of the 45 -year- old Petten nuclear reactor in the Netherlands, while simultaneously starting the experimental Thorium PALLAS project in Patten as an alternative uranium fuel source, will certainly comprise global medical isotope delivery further. In this mini-review the history and discovery of molybdenum, its use and production, waste problems and alternative cyclotron medical isotope production are discussed.

\section{Discovery and History of Mo-99}

In ancient times a number of substances were collectively known by the Greek word "molybdos" meaning lead-like. Molybdenite (M0S2), the most abundant molybdenum-containing mineral was in this class along with lead, galena, graphite and others. Molybdenum has been found in a 14th century Japanese sword as an alloying element [6]. In 1768, the Swedish scientist Carl Wilhelm Scheele determined that molybdenite was a sulfise compound of an as yet unidentified element, by decomposing it in hot nitric acid and heating the product in air to yield a white oxide powder. In 1782, at Scheele's suggestion, Peter Jacob Hjelm chemically reduced the oxide with carbon obtaining a dark powder that he named "molybdenum". Molybdenum remained mainly a laboratory curiosity until late in the 19th century, when technology for the extraction of commercial quantities became practical. Experiments with steel demonstrated that molybdenum could effictively replace tungsten in many steel alloys.

This change brought weight benefits, since the atomic weight of tungsten is nearly twice that of molybdenum. In 1891, the French 
company Scneider \& Co first used molybdenum as an alloying element in armoured plate steel. Demand for alloys steel during World War 1 caused tungsten demand to soar, severely straining its supply. The tungsten shortage accelarated molybdenum substitution in many hard and impact-resistant tungsten steels. This increase in molybdenum demand spurred an intensive search for new sources of supply, culminating with the development of the massive Climax deposit in Colorado, U.S.A. and its startup in 1918.

After the war reductions in alloy steel demand triggered intense research efforts to develop new civilian applications for molybdenum and a number of new low-alloy molybdenum automotive steels were soon tested and accepted. In the 1930's, researchers determined the proper temperature ranges to forge and heat treat molybdenum-bearing-high speed steels, a breakthrough that opened large new markets to molybdenum.

Researchers eventually developed a full understanding of how molybdenum imparts its many cost-effective benefits as an alloying element to steels and other systems. By the end of the 1930's molybdenum was a widely accepted technical material. The end of World War 2 in 1945 once again brought increased research investment to develop new civil applications, and the post-war reconstruction of the World provided additional markets for molybdenum-containing structural steels. Steels and cast iron still compromise the single biggest market segment, but molybdenum has also proven to be invaluable in superalloys, nickel base alloys, lubricants, chemicals, Electronics, superconductors and many other applications, as e.g., a medical diagnostic isotope [6].

\section{Production and use of Molybdenum-99/Thechnetium- $99 \mathrm{~m}$}

In 2009, U.S. Congress mandated the study of medical isotope production without highly enriched uranium (HEU)-[7,8]. The study mandate included the medical isotopes molybdenum 99, iodine 131, xenon 133 and other radioactive materials used to produce radiopharmaceuticals for diagnostic and therapeutic procedures for research and development. The authoring committee of the study determined that for the purposes of addressing the statement of task force for this study, it is sufficient to focus on the production of the medical isotope molybdenum 99 (Mo-99). This is so because:

a) The decay product of Mo-99, technetiun-99m (Tc-99m) is used in about two thirds of all diagnostic medical isotope procedures in the United States.

b) Between 95 and 98 percent of Mo-99 is currently being produced using highly enriched uranium (HEU) targets (NNSA and ANSTO,2007), which was the major concern of Congress when it mandates this study.

c) Other medical isotopes such as iodine-131(I-131) and Xenox-133 (Xe-133) are by-products of the Mo-99 production process and will be sufficiently available if Mo-99 is availaible.

d) These other medical isotopes are not being recovered for sale by all Mo99 producers because they can be more cheaply produced from other sources (e.g., Russian English Venture in Isotope Supply (REVISS) sells Russian-produced isotopes).

Point $\mathrm{c}$ deserves additional elaboration. The fission of uranium-235 ( U-235) produces a spectrum of fission products including Mo-99, I-131 and Xe-133.These fission products are produced in the same proportions to each other, whether HEU (highly enriched uranium) or low -enriched uranium (LEU) targets are used. All of these isotopes can be recovered when the targets are processed to obtain Mo-99.

\section{Molybdenum-99 use in Nuclear Medicine}

The decay product of Mo-99,Tc-99m is the workhorse isotope in nuclear medicine for diagnostic imaging. Tc-99m is used for the detection of disease and for the study of organ structure and function. Tc- $99 \mathrm{~m}$ is especially useful for nuclear medicine procedures because it can be chemically incorporated into small molecule ligands and proteins that concentrate in specific organs or tissues when injected in the body. The isotope has a half-life of 6 hours and emits $140 \mathrm{KeV}$ photons when it decays to Tc-99, a radio-isotope with about a 214.000 year hal-life. This photon energy is ideally suited for efficiënt detection by scintillation instruments such as gamma cameras, and nowadays also by CT or SPECT (specific position emission tomography) are analyzed to produce detailed structural and functional images.

Tc-99m is currently produced through a multistep process that begins with the neutron irradiation of fissile U-235 contained in HEU or LEU targets in a nuclear reactor. This irradiation causes U-235 to fission and produces Mo-99 and many other fission products. Following irradiation, the targets are chemically processed to separate Mo-99 from other fission products, including I-131 and Xe-133, which can be recovered separately, if desired. The separated Mo-99, which is contained in a solution, is absorbed onto an alumina column (AL203) that is contained in cylinders that are about the diameter of a large pencil. The columns are shipped to radio pharmacies and hospitals in radiation-shielded cartridges known as "technetium generators". The Mo-99 in the generators decays with about a 66-hour half-life to Tc-99m. The Tc-99m is typically recovered by passing a saline solution through the alumina column in the generator, a process known as "eluting the generator". The saline removes the Tc- $99 \mathrm{~m}$ but leaves the Mo-99 in place. A technetium generator can be eluted several times a day before it needs to be replaced by a fresh generator. After 1 week Tc-99m can be contaminated with M0 99 from the column.

There are numerous Tc-99m kits for producing radiopharmaceuticals to examine the brain, kidney, heart, bone liver, endocrine glands and lung. Kits are composed of all the required chemicals (e.g., the pharmaceutical agent, chelating compound and saline solution) for formulating the radiopharmaceutical to which Tc- $99 \mathrm{~m}$ is added. After it is replaced the old generator may be used for research that does not involve human subjects. Because of its relatively short half-life (66 hours) Mo-99 cannot be stockpiled for use. It must be made on a weekly or more frequent basis to ensure continuous availability. The processes for producing Mo-99 are tightly 
scheduled and highly time dependent. An interruption at any point in the production, transport, or delivery of Mo-99 or technetium generators can have substantial impacts on patient care.

\section{M0-99 Production Process}

There are two approaches for producing the medical isotope Mo-99; fission of U-235, which produces Mo-99 and other medical important isotopes such as I-131 and Xe-133 and the neutron capture method by Mo-98 to produce Mo-99. The committee dismissed neutron capture as a viable process for producing Mo-99 in the quantities needed to meet U.S. or global demand for Mo-99 because of its inefficiencies. However, this process can be used to make smaller quantities of Mo-99.

\section{HEU or LEU targets?}

These 3 processes apply whether Mo-99 is produced from HEU or LEU targets:

a) Fabrication of uranium targets.

b) Irradiation of targets in a nuclear reactor.

c) Dissolution of the uranium target and recovery and purification of Mo-99.

The equipment used to produce Mo-99 is small. The process equipment is "bench scale" compared to most industrial chemical processing applications and has the size of a large dining room table. Of course, this processing equipment must be operated inside large and heavily radiation-shielded facilities because the irradiated targets that contain Mo-99 are highly radioactive.

\section{Fabrication of Uranium Targets}

The target used for Mo-99 production is a material containing uranium-235 that is designed to be irradiated in a nuclear reactor. The target is designed to meet several requirements. First, it must be properly sized to fit in the irradiation position inside the reactor. Second, it must contain a sufficient amount of U-235 to produce the required amount of Mo-99 when it is irradiated. Third, it must have good heat transfer properties to prevent overheating, which could result in target failure during irradiation. Fourth, the target must provide a barrier to the release of radioactive products, especially fusion gases, during and after irradiation. Fifth, the target material must be compatible with the chemical processing steps that will be used to recover and purify Mo-99 after the target is irradiated.

To meet these criteria, targets are fabricated in a wide variety of shapes and compositions to meet the needs of individual Mo99 producers. Targets may be shaped as plates, pins or cylinders. Target compositions include uranium metal, uranium oxides, and alloys of uranium, nearly always with aluminium. Metallic targets are typically encapsulated in aluminium or stainless steel to protect the chemically reactive uranium metal or alloy and to contain the fission products produced during irradiation. The encapsulation is referred to as the "target cladding" Sometimes an intermediate barrier material such as aluminium or nickel is used to separate the cladding from the U-235 material.

\section{Irradiation of Targets in a Nuclear Reactor}

Mo-99 is produced in the uranium-bearing targets by irradiating them with thermal neutrons, which can cause them to fission. The fission of the U-235 nucleus produces two but sometimes three lower-mass nuclei referred as fission fragments. Approximately 6 percent of these fission fragments are Mo-99 atoms. Nuclear reactors provide an efficient source of thermal neutrons for Mo-99 production. This is why all major Mo-99 producers irradiate their targets in nuclear reactors. The amount of Mo-99 produced in a target is a function of irradiation time, the thermal neutron fission cross section for U-235, the thermal neutron flux on the target, the mass of U-235 in the target and the half-life of Mo-99. For typical reactor thermal neutron fluxes on the order 10 up to 14 detach neutrons per square centimeter per second irradiation times of about 5-7 days are required to achieve near-maximum Mo-99 production in the targets. Beyond these irradiation times, the amount of Mo99 produced in the targets approximately balances the amount of Mo-99 being lost to radioactive decay, so further irradiation is not productive. Even at maximum production, only about 3 percent of the U-235 in the target is typically consumed. The remaining U-235 along with the other fission products and target materials are treated as waste.

\section{Dissolution and Mo-99 Recovery}

Once the targets are removed from the reactor, they are cooled in water typically for half a day or less before being transported to the processing facility in shielded casks. Once at the processing facility, the targets are placed into hot cells for chemical processing. Processing is carried out quickly to recover the Mo-99 to minimize further losses from radioactive decay. About 1 percent of the Mo-99 produced in the target is lost to radioactive decay every hour after irradiation. The apparatus in the hot cell used to process the targets and recover the Mo-99 consists of a container for dissolving the targets which is connected to tubing and columns for subsequent chemical separations to isolate Mo-99. The components can be easily replaced or reconfigured by a human operator using remote manipulators. The most expensive part of the separation facilities are the hot cells themselves. Hot facilities can cost tens of millions of dollars to construct. There are two general approaches for chemically processing targets to recover Mo-99; Alkaline dissolution and acidic dissolution. The processes can be used on both HEU and LEU targets.

\section{Alkaline Dissolution Process}

Alkaline dissolution is generally used for targets that contain aluminum. A sodium hydroxide $(\mathrm{NAOH})$ solution is used to dissolve the entire target including the aluminum cladding and the aluminum alloy "meat" of the sandwich target model. Dissolution produces a sodium aluminate $\left(\mathrm{NaAlO}_{2}\right)$ solution containing sodium molybdate $\left(\mathrm{Na}_{2} \mathrm{MoO}_{4}\right)$ along with small amounts of fission products and plutonium $(\mathrm{Pu})$ and a solid oxide hydrated oxide residue. Hydrogen gas is evolved during dissolution. The solid residue contains uranium and most of the fission products except the alkali metals, iodine, fission gases, alkaline earths, and the elements that can act 
as either an acid and or base such as molybdenum and aluminum. The short lived fission gases (e.g., Xe-133) can be collected for sale or stored for decay, and I-131 can also be separated for sale if desired.

The solution is recovered by filtering to remove suspended solids, typically purified by ion-exchange, and passed through a column of alumina, that preferentially adsorbs the molybdate MbO42)ion. Mo-99 recovery yield from the solution typically exceeds 85-90 percent. The sorbet molybdate is typically washed with a dilute ammonium hydroxide $(\mathrm{NH} 4 \mathrm{OH})$ solution and then removed from the column using a concentrated saline or ammonium hydroxide solution. Mo-99 is recovered as a highly pure product.

\section{Acid Dissolution and Molybdenum Separations Process}

Acid dissolution is generally used for uranium metal and uranium oxide targets. It is currently used by only one major producer, MDS Nordion. In contrast to the alkalie dissolution process, only the uranium metal or oxide is processed: the uranium target meat is physically separated or leached from the target cladding and then solved in nitric acid. A nitrate solution containing uranium, molybdenum, and all other fission products (except volatile gases such as iodine, Xe- 133, Krypton-85 and nitrogen -oxides) is formed. Additional processing steps are required to recover pure molybdenum. Molybdenum can be separated from the nitrate solution by any of several separation processes. Typical separation processes include adsorption of the molybdenum on ion exchange resins and solvent extractions.Mo-99 recovery yields from these separation processes exceed 85 to 90 percent The adsorbed or extracted molybdenum is washed with an appropriate solution to remove residual fission products and uranium. The wash solution becomes waste. The adsorbed or extract molybdenum is then removed from the separation medium using an appropriate solution and receives a highly purified Mo-99 product.

\section{Waste Management}

Waste management is similar for both the alkaline and dissolution processes. In the alkaline process, the sodium aluminate and dissolved or suspected products that pass through the alumina are combined with the other fission product wastes and precipitate oxide residues. This waste is stored temporarily either as, or put in a solid form (e.g,in cement).The waste stream from the acid dissolution process includes the separate cladding and liquid waste from the Mo-99 separation or extraction processes. This liquid waste can be stored in tanks or mixed with cement to immobolize it. Most of these processes wastes are stored at producers'sites or are transported to offsite storage facilities. Approximately 97 percent of the uranium originally present in the target ends up in the process waste. Consequently the accumulating waste from Mo-99 production contains substantially quantities of HEU. Worldwide tens of kilograms of this HEU waste are accumulating annually from Mo-99 production. This HEU could be recovered for reuse, but currently no producer has active plans to do so, because it is less costly to purchase fresh HEU. Additionally, few Mo-99 producers download their HEU waste (by mixing it with natural or depleted uranium) to convert it to LEU. In 2018, the Petten research reactor will start the already 2006 planned new High Flux Reactor for converting HEU to LEU [9]. While HEU is suitable to proliferate nuclear weapons, LEU is not.

\section{Process Trade-offs}

Both the alkaline and acidic dissolution processes have been proven to be effective through many years of use with HEU targets. Both dissolution processes might be adapted to LEU processing. However, each of these processes have inherent advantages and disadvantages. For example, alkaline processing produces very pure Mo-99, solid waste that is suitable for storage, and fission gases that can be readily isolated for sale or for storage to allow for decay. On the other hand, relative to the acid process, alkaline processing produces larger volumes of processing solutions, it can require more time than the acidic process for target dissolution, and Mo-99 yields can be lower because some molybdenum may be incorporated into the solid residue. Additionally, hydrogen gas is produced in the alkaline process, which requires additional safety procedures.

Acidic processing, in contrast, generally requires shorter processing times, produce smaller volumes of processing waste, and results in slightly higher Mo-99 yields. On the other hand, additional steps have to be carried out to separate the Mo-99 from the processing solutions, and there needs to be a separate process for handling the treatment of the nitrogen oxide gases given off from the process. Armed with this basic knowledge, provided by the National Academy of Sciences and Sciences Engineering Medicine $[2,3]$, new alternatives sources and strategies can be discussed now.

\section{Alternative Sources and Strategies}

Particle physics and CERN (Conseil European pour la Recherche Nuclearize) in particular has made major contributions to medicine. Key to this, in addition to detectors for diagnosis and medical imaging, is accelerator technology. A new high-energy proton therapy centre in Nice, France, which has its roots in a CERN project is about to treat its first patients, offering more precise tumor treatment than is possible with conventional X-rays. With global demand for isotopes such as technetium-99m growing and many reactors reaching the end of their operational lifetimes, CERN has recently launched a project called MEDICIS to produce isotopes from high -energy proton beams. Meanwhile Brookhaven National Laboratory in the U.S. has undergone a series of upgrades to boost its long-running isotopes programmed and TRIUMP in Canada is pursuing isotopes for the rapidly growing field of targeted alpha therapy [10].

TRIUMP announced in 2015 in collaboration with an Argentinian research group that it found an alternative for leaking, high maintenance costs, waste toxic nuclear plants by producing medical isotopes by using cyclotron-based production of isotopes [11]. Researchers of the University of British Colombia (BC) claimed in 2015 that their cyclotron has produced enough isotopes in six hours to conduct about 500 scans. Cyclotron based production of Tc-99m would provide a solution to shift away from reactor-based production of isotopes and secure supply for at least Canadian patients, the researchers say $[11,12]$. MURR (Missouri University Re- 
search Reactor Center) created also an $16 \mathrm{MeV}$ cyclotron to create commercial products for medical imaging. Delivery is aimed the University of Missouri and the region for their implementation and research use $[5,13]$. However, it is clear that cyclotron production of medical isotopes serves only local and domestic needs. Its limited production cannot supply the global medical isotope shortage at this moment as an interesting UK House of Parliaments, Post Note shows [14]. A cyclotron generator has to deliver the Technetium-99m the same day because of the short half-life of Tc-99m (6 hours).Five hundred samples a day is a limited capacity for a global market of 40-50 million investigations annually. In contrast, a single Mo-99 generator lasts 1-2 weeks, as discussed before and can be used multiple times a day (14).It is not known how the costs of cyclotron-produced Tc-99m would compare to that of Mo-99 generators, though some estimate it would be more expensive [15].

\section{PALLAS}

PALLAS aims to realize a state of the art multifunctional nuclear reactor, which is suitable for mainly producing medical technology and conducting research. This reactor will replace the current High Flux Reactor (HFR) in Patentee Netherlands, which has been in operation for over fifty years and is approaching the end of its economic life. In January 2018, constructors for PALLAS were selected $[16,17]$. From 2025 onwards PALLAS, one of the world's leading suppliers of medical isotopes, will play a crucial role in the supply chain for radiopharmaceutical products worldwide and in nuclear technology research. In European hospitals 70\% of isotopes used for diagnostic procedures (e.g., cancer, cardiovascular diseases) and for treatment, originate from the current HFR in Patten. Globally, this percentage is $30 \%$ and in the Netherlands, it is as high as 80\%. The PALLAS organization was founded on Dec. 16,2013, with the purpose to design and construct the reactor, to obtain the necessary licenses develop a solid business case, and to arrange private financing for the construction and commissioning phase of the PALLAS reactor. In Argentina, the Anucha 3 Nuclear Power Plant will be built in 2018. In Brazil, the construction work of the Angra 3 Nuclear Power Plant will also be restored, following the announcement of a new electronuclear partner in 2018. In Mexico there are also some updates in the nuclear industry [18]. Saying nobody would invent closing a Canadian reactor and starting construction work in 4 of the 7 available reactors ,all in 2018,is merely an understatement.

\section{Conclusion}

Unfortunate coincidence due to definite closing of the Chalk River reactor in Canada and multiple extensive nuclear construction works in the other 6 nuclear plants in 2018 will comprise medical isotope supply severely in the next decade. Cyclotron production of technetium-99m can only meet local and domestic needs and is not able to solve the global shortage of medical isotopes and radiopharmaceuticals. Production of isotopes from high-energy proton beams in the CERN's MEDICIS project is still in a developmental phase and offers no comfort now. This global molybdene shortage will have substantial impacts on patient care, at least until 2025 when the PALLAS reactor in Patentee Netherlands, must be ready.

\section{References}

1. Tollefson J (2016) Reactor shutdown threatens world's medical isotope supply. Nature.

2. Riya V (2017) New Report Warns of Potential Supply Shortage of the Medical Isotopes Molybdenum 99 and Technetium 99m in the U.S.A. Newsletter 2017, The National Academics of Sciences-EngineeringMedicine.

3. (2016) Sciences Engineering Medicine. Molybdenum-99 for Medical Imaging, Chapter 3, Global Production of Molybdenum-99 and Future Prospects. Nat Acad Press p.51-246.

4. Varney S (2018) Inside the Global Race to Deliver Moly-99. New York Times p. 5.

5. (2017) General Atomics Press Releases. University of Missouri Research Reactor Files for NRC Approval to Start US. Production of Medical Isotopes. MURR: Colombia, MO.

6. IMOA: International Molybdenum Association: London, UK.

7. (2009) Medical Isotope Production without Highly Enriched Uranium. National Research Council (U.S.) Committee on Medical Isotope Production without Highly Enriched Uranium, Medical Isotope Production without Highly Enriched Uranium, Washington DC, National Academic Press US 2, Molybdenum-99/Technetium-99m, Production and use.

8. (2016) Molybdenum-99 for Medical Imaging, Chapter 3; Global Production of Molybdenum-99 and Future Prospects. The National Academic of Sciences Engineering Medicine.

9. (2006) European Nuclear Society. e-news, Petten research reactor converts to low-enriched fuel p. 13.

10. (2016) Cern Courier-digital edition.

11. Crawford T (2015) Breakthrough UBC research finds cleaner,safer source of medical isotopes than nuclear power plants. Vancouver Sun.

12. Longheed T (2013) Cyclotron production of medical isotopes scales up. CMAJ 185(11): 947.

13. MURR. Radioisotopes and Radiochemical.

14. (2017) Houses of Parliament, UK. Parliamentary Office of Science \& Technology. Supply of Medical Radioisotopes 558.

15.Zimmerman RG (2010) Molybdenum-99 supply shortage:is cyclotronproduced Technetium-99m a realistic alternative? Tijdschr Nucl Geneesk 32(4): 617-620.

16. (2018) PALLAS Has Selected Consortium INVAP, Mobilis and Croon Wolters \&Dros Invap fort he Design and Construction of the PALLAS Reactor Mobilis/TBI.

17. (2018) PALLAS design and construction contract awarded. World Nuclear News (WNN).

18. (2018) NISLA, Summit Argentina. Third Annual Nuclear Industry Summit Latin America. 
(C) (P) This work is licensed under Creative Submission Link: https://biomedres.us/submit-manuscript.php

Assets of Publishing with us
REMEDICAL $\quad \begin{aligned} & \text { Global archiving of articles } \\ & \text { - Immediate, unrestricted online access }\end{aligned}$

